\title{
Clinical and radiological features of novel coronavirus pneumonia
}

\author{
Qiuting Zheng ${ }^{\mathrm{a}}$, Yibo Lu ${ }^{\mathrm{b}}$, Fleming Lure ${ }^{\mathrm{c}, \mathrm{d}, *}$, Stefan Jaeger ${ }^{\mathrm{e}}$ and Puxuan Lu ${ }^{\mathrm{a}, *}$ \\ a Department of Medical Imaging, Shenzhen Center for Chronic Disease Control, \\ Guangdong Shenzhen 518020, China \\ ${ }^{\mathrm{b}}$ Department of Medical Imaging, The Fourth People's Hospital of Nanning, \\ Guangxi Nanning 530023, China \\ ${ }^{\mathrm{c}}$ MS Technologies, 10110 Molecular Dr., Suite 305, Rockville, MD 20850, USA \\ d Shenzhen Zhiying Medical Co., Ltd, Guangdong Shenzhen 518020, China \\ e National Library of Medicine, National Institutes of Health, 8600 Rockville Pike, \\ Bethesda, MD 20894, USA
}

Received 7 April 2020

Revised 18 April 2020

Accepted 5 May 2020

\begin{abstract}
Recently, COVID-19 has spread in more than 100 countries and regions around the world, raising grave global concerns. COVID-19 transmits mainly through respiratory droplets and close contacts, causing cluster infections. The symptoms are dominantly fever, fatigue, and dry cough, and can be complicated with tiredness, sore throat, and headache. A few patients have symptoms such as stuffy nose, runny nose, and diarrhea. The severe disease can progress rapidly into the acute respiratory distress syndrome (ARDS). Reverse transcription polymerase chain reaction (RT-PCR) and Next-generation sequencing (NGS) are the gold standard for diagnosing COVID-19. Chest imaging is used for cross validation. Chest CT is highly recommended as the preferred imaging diagnosis method for COVID-19 due to its high density and high spatial resolution. The common CT manifestation of COVID-19 includes multiple segmental ground glass opacities (GGOs) distributed dominantly in extrapulmonary/subpleural zones and along bronchovascular bundles with crazy paving sign and interlobular septal thickening and consolidation. Pleural effusion or mediastinal lymphadenopathy is rarely seen. In CT imaging, COVID-19 manifests differently in its various stages including the early stage, the progression (consolidation) stage, and the absorption stage. In its early stage, it manifests as scattered flaky GGOs in various sizes, dominated by peripheral pulmonary zone/subpleural distributions. In the progression state, GGOs increase in number and/or size, and lung consolidations may become visible. The main manifestation in the absorption stage is interstitial change of both lungs, such as fibrous cords and reticular opacities. Differentiation between COVID-19 pneumonia and other viral pneumonias are also analyzed. Thus, CT examination can help reduce false negatives of nucleic acid tests.
\end{abstract}

Keywords: 2019-novel Coronaviruses (2019-nCoV), SARS-CoV-2, COVID-19, Computed Tomography (CT), ground glass opacity (GGO), differentiation of pneumonia

\section{Background and current situation of the epidemics}

Since December 2019, an epidemic of pneumonia of unknown cause has spread rapidly to the whole Hubei Province, the whole country, and multiple regions and countries around the world. The pathogen

\footnotetext{
*Corresponding author: Fleming Lure, MS Technologies, 10110 Molecular Dr., Suite 305, Rockville, MD 20850, USA; Shenzhen Zhiying Medical Co., Ltd, Guangdong Shenzhen 518020, China. E-mail: f.lure@ hotmail.com; Puxuan Lu, Department of Medical Imaging, Shenzhen Center for Chronic Disease Control, Guangdong Shenzhen 518020, China. E-mail: lupuxuan@126.com.
} 
of this pneumonia with unknown cause was isolated successfully by Chinese scientists on January 7th, 2020 and confirmed to be a newly-discovered novel coronavirus through whole-genome sequencing. The Coronaviridae Study Group (CSG) of the International Committee on Taxonomy of Viruses, World Health Organization (WHO), has assessed the placement of the human pathogen, tentatively named 2019-nCoV, within the Coronaviridae. CSG recognizes this virus as forming a sister clade to the prototype human and bat severe acute respiratory syndrome coronaviruses (SARS-CoVs) of the species Severe acute respiratory syndrome-related coronavirus, and designates it as SARS-CoV-2. Sequencing was completed for the virus on January 10th, 2020. China then shared the data globally (https://www.gisaid.org/), and shortly after a diagnostic method based on fluorescence quantitative RT-PCR was developed for 2019-nCov [1]. The epidemic of novel coronavirus pneumonia in China has become a "Public Health Emergency of International Concern (PHEIC)", as declared by Tedros Adhanom Ghebreyesus, director general of WHO, in Geneva, on January 30th, 2020. The National Health Commission issued Announcement No. 1 on January 20th, 2020, listing novel coronavirus pneumonia as one of the Statutory Class-B Infectious Diseases, for which Class-A Measures would be adopted [2]. On February 11th, 2020, WHO named novel coronavirus pneumonia as "COVID-19", with CO standing for corona, VI for virus, and D for disease.

As of April 5th, 2020, there are 1,299 existing confirmed cases (including 265 severe cases), 77,078 accumulative cases cured and discharged, 3,331 accumulative deaths, 81,708 accumulative confirmed cases, and 88 existing suspected cases, as reported by 31 provinces (autonomous regions or municipalities directly under the central government) and Xinjiang Production and Construction Corps in China. Globally, the epidemic is rampant in the Western Pacific, Europe, Southeastern Asia, the Middle East, the Americas, and Africa, manifesting most severely in USA, Spain, and Italy, while the number of infected people continues to rise. The global impact by the epidemic is far greater than that induced by SARS in 2003 [3].

\section{Etiological features and pathogenic mechanism}

The literature has attributed the outbreak of the COVID-19 Pneumonia to a place called Huanan Seafood Market in Wuhan City, Hubei Province, China, so far, where live animals such as poultry, bats, marmots and other wild animals are sold, suggesting the possible transmission of pathogens from animals to humans [4]. The present whole-genome phylogenetic analysis on 2019-nCoV shows that it has a much closer phylogenetic relationship to the SARS-like coronaviruses bat-SL-CoV ZC45 and bat-SL-CoV ZXC21 from Rhinolophus Sinicus (a species of Chinese Horseshoe Bat) [5-7]. Therefore, bats are considered the possible main host of 2019-nCoV [8]. It has not been defined, however, whether 2019-nCoV pneumonia is transmitted through Rhinolophus Sinicus directly or via an intermediate host. Most scholars believe that Rhinolophus Sinicus is the most primitive host of the 2019-nCoV, and that the virus might be spread to humans through a yet unknown animal host, possibly pangolin [9, 10]. Recently, 149 mutational sites in $2019-\mathrm{nCoV}$ have been detected, as suggested by the most recent Chinese research. The virus has evolved into two subtypes, L- and S-subtype, with the latter having stronger aggressiveness and infectiousness [11].

The results of Coronavirus gene sequencing performed by Brazilian researchers for the first confirmed case in Brazil, on February 26th, showed three differences in the Novel Coronavirus (Brazil/SPBR1/2020) compared with the virus gene (Hu-1 Reference Strain) published in Wuhan; that is to say, mutations might have occurred in the virus during its transmission [12].

Corona viruses belong to the subfamily of Ortho coronavirus under the family of Coronaviridae and catalog of Nidovirales. This subfamily comprises four attributes including Alpha-coronavirus $(\alpha)$, Beta-coronavirus $(\beta)$, Gamma-coronavirus $(\gamma)$, and Delta-coronavirus $(\delta)$. Attributes $\alpha$ and $\beta$ tend 
to infect mammals, and contain seven species of coronavirus causing disease in humans, including HCoV-OC43, HCoV-229E, SARS-CoV, HCoV-NL63, HCoV-HKU1, MERS-CoV, and SARS-CoV-2 (2019-nCoV), while attributes $\gamma$ and $\delta$ infect mainly birds [13]. 2019-nCoV, with a genome structure typical of coronavirus, is a positive single-strand RNA virus particle capsulated in a diameter of about $60-140 \mathrm{~nm}$ and a size of $30 \mathrm{~kb}$. With spike spines on its capsule, the whole virus looks like a corona [14-16].

The genome of coronavirus usually encodes four structural proteins, including spike protein (S), membrane protein $(\mathrm{M})$, envelope protein $(\mathrm{E})$, and nucleocapsid protein $(\mathrm{N})$. Some coronaviruses of attribute $\beta$ also encode hemagglutinin esterase protein (HE). S protein mediates the attachment of the virus to a receptor on a cellular surface and is one of the key factors achieving effective interpersonal transmission. Protein S, belonging to Type-I transmembrane glycoprotein, consists of two domains: a receptor-binding subunit $(\mathrm{S} 1)$ and a membrane fusion subunit $(\mathrm{S} 2)$. In the process of virus invasion, S1 is responsible for binding with the receptor on the surface of the host cell followed by viral attachment, and S2 is responsible for fusing the cell membrane of the host and viral envelope, making the virus genome enter the host cell to form a stable binding compound. The whole process of infection consists of four steps: adsorption invasion, gene synthesis, packaging of the mature virus, and virus release [17, 18]. Through the pathway of S protein binding ACE2 receptor in human cells, 2019-nCoV constitutes a major risk for public health due to human transmission [6]. In addition, ACE2 is mainly situated at alveolar Type II epithelial cells in the lower lungs, so 2019-nCoV is more likely to cause severe diseases such as pneumonia.

\section{Epidemiological features}

Most of the early patients infected with COVID-19 pneumonia were in Wuhan, indicating a local outbreak [1]. Later, most of the patients had been to Wuhan or in close contact with patients coming from Wuhan [19-21]. Meanwhile, confirmed cases have appeared in other regions of China and have been reported in many countries and regions outside of China [22]. Infection of medical staff and family clusters has shown that SARS-CoV-2 spreads in population clusters [23] with stronger infectiousness than SARS-CoV or MERS-CoV [19], and that the epidemic has developed into community transmission. The epidemic has expanded rapidly and spread from Hubei Province to other areas of China with the immigration of infected people, while the number of cases increased gradually all over the world.

The main source of infection is the population of COVID-19 patients, including asymptomatic infected people. Identification of the transmission chain and subsequent tracing of the contacts become more complex when several infected people seem asymptomatic or only mildly symptomatic [24]. The main routes of transmission include respiratory droplets and close contact. Transmission via aerosols seems possible in case of prolonged exposure to aerosols in high concentration in a relatively closed environment. SARS-CoV-2 has been isolated from urine and feces of patients in multiple regions; therefore, transmission via aerosol or contact due to environmental contamination should be considered [14, 20]. Mother-to-child transmission need to be confirmed, as well as other transmissions [10]. The latent period lasts 1-14 days, mostly 3-7 days. The longest reported latent period is 24 days [20], although in an individual case. People are generally susceptible, and patients are concentrated in a population aged between 30 and 79 years [19] and are correlated with exposure to viral load. Conditions after infection are more serious in the older patients and those with underlying diseases, while severe diseases seem rare among children and infants $[22,25]$. 


\section{Clinical manifestation and laboratory examination}

The symptoms of SARS-CoV-2 infection seem to be non-specific, as far as clinical manifestations are being concerned. They may be very similar to influenza and manifest mainly in fever, fatigue, dry cough, sore throat, headache, and occasionally nasal congestion, runny nose, and diarrhea in a few patients. Clinically, cases can be classified into mild, common, severe, and critical. Dyspnea and/or hypoxemia occur(s) usually in severe cases within one week. Serious cases can progress rapidly into acute respiratory distress syndrome (ARDS), septic shock, refractory metabolic acidosis, and coagulation dysfunction. It is noteworthy that patients with severe or critical diseases might manifest only moderate to low fever, or even no obvious fever during the course of the disease. Patients with mild disease may manifest only low fever and mild fatigue without manifestations of pulmonary inflammation and can recover after one week in most cases.

RNA, the hereditary substance of the virus, may become detectable post systemic infection of SARS-CoV-2. Nucleic acid detection aims to find RNA of SARS-CoV-2 in samples from the patient, so it is also the "Golden Standard" and an important approach in clinical diagnosis. There are mainly two methods in detecting nucleic acids of SARS-CoV-2, including NGS (Next-generation sequencing) and reverse transcription polymerase chain reaction (RT-PCR) [26]. NGS, the next generation of sequencing technology, was the first method that succeeded in detecting the new pathogen at the initial stage of the epidemic and defined soon the sequences of nucleic acids of SARS-CoV-2. The simplest and fastest strategy for detection will be undoubtedly the targeted fluorescence quantitative RT-PCR using primer probes designed for the conserved domains of the nucleic acid sequence that have been defined for the virus. This method involves a proliferation of specific RNA sequences in the sample post to their reverse transcription. Theoretically, the quantity of genome segments of the virus post to each amplification will be multiplied, and the visual detection will become feasible when segments of the targeted genes reach a certain number after more than 30 amplifications. Targets detected for nucleic acids of SARS-CoV-2 consist of three conserved sequences in the viral genome, including open reading frame 1ab (ORF1ab), nucleocapsid protein (N), and Envelope gene [27].

According to the Laboratory Guidelines for Novel Coronavirus Infections issued by the China Health Commission [28], the prerequisite for a laboratory confirmation of a positive case should be the positiveness of real-time fluorescence RT-PCR results in two specific targeted genes including ORF1ab and gene N, or single-target positiveness of RT-PCR either in two types of specimens simultaneously or in two separately-sampled specimens of the same category. Re-sampling and re-test are needed, in case of a positiveness of a single target. The test should be performed at least for ORF1ab region, the most conserved and the most specific region of 2019-nCoV, if only one target gene is detectable because of limited availability of the selected laboratory kit. COVID-19 cannot be ruled out, even if there is a negative result. Factors inducing false negative should be excluded, such as poor quality of the specimen (e.g., specimen from respiratory tract such as oropharynx), specimens collected too early or too late, incorrect conservation, transportation or treatment, reasons of the technology per se (e.g., viral mutation, PCR inhibition) [29].

The leukocyte count in peripheral blood is normal or decreased in early stage, with a low lymphocyte count, especially low numbers of $\mathrm{T}$ lymphocytes, which are in an over-activated state that can cause severe immune damage in patients [30]. C-reactive protein (CRP) and ESR increase in most patients, and procalcitonin remains normal. In quite a few patients, increase of liver enzymes, muscle enzymes, and myoglobin are observed. In severe cases, D-dimer increases and lymphocytes in peripheral blood decrease progressively. SARS-CoV-2 can be positive in specimens including nasopharynx swab, sputum, secretion frp, secretion of lower respiratory tract, blood, urine, and feces. Importantly, symptoms last longer in cases of SARS-CoV-2 than in most cases of non-complicated influenza [31, 32]. Despite non-typical symptoms occurring in a few cases, fever is still the typical symptom of a 
SARS-CoV-2 infection. Patients with underlying chronic diseases generally have prolonged courses, severer conditions, more rapid progressions, and worse prognoses [21, 31].

\section{Pathological and radiological manifestations}

The autopsy of the first death due to COVID-19 in China showed obvious lung injuries, with patchy grayish lesions and dark red hemorrhage discernable by the naked eye. The texture of the lung tissue became hard, losing the sponginess inherent to lungs. A large amount of thick secretions could be seen escaping from the section of alveoli, and fiber cords were observable [33].

Histological examination: under the light microscope, pulmonary interstitial blood vessels were congested and edematous, with inflammatory infiltration of lymphocytes and monocytes as well as clear thrombosis in the vessels. Serous fluid, fibrin exudate, and formation of hyaline membrane were observed in the alveolus cavities. The exudate cells were mainly monocytes and macrophages, and multinucleated giant cells were also common. In addition, atypical enlarged alveolar cells could be seen, in which the atypical enlarged alveolar cells had large nuclei, amphiphilic cytoplasmic granules and obvious nucleoli, showing viral cytopathic-like changes [30]. Some alveolar epithelial cells were exfoliated, with inclusion bodies inside. Partial alveolar exudate organization and interstitial fibrosis occurs. Local hemorrhage, necrosis, and hemorrhagic infarction might occur in lung tissue. Part of the mucous epithelium of intrapulmonary bronchi was exfoliated, with mucus and mucus thrombus observable in the lumen. A few alveoli were overinflated, with broken alveoli septum or formation of cysts. Under an electron microscope, coronavirus particles could be seen in the cytoplasm of bronchial epithelial cells and Type II alveolar epithelial cells. Immunohistochemistry staining proved the presence of SARS-CoV-2 antigen in some of the alveolar epithelial cells and macrophages, and SARS-CoV-2 nucleic acid was positive as shown by RT-PCR [14]. Local pathological changes suggested a high similarity of COVID-19 with SARS-CoV and MERS-CoV infection.

DR imaging is a convenient and fast examination method for patients with lung diseases, but low sensitivity and specificity due to the influence of an overlap of anatomic structure on the observation of lesions make it easy to miss the image manifestation of COVID-19. COVID-19 usually has no abnormal change or manifests as bronchitis in the early stage. In the progression stage, it may manifest limited or multiple segmental patchy opacities in the middle and peripheral zones/subpleural areas of both lungs. In severe patients, multiple consolidations and ground-glass opacities (GGO) are seen in both lungs, and some of them are fused into large consolidations, with a small amount of pleural effusion or pleural thickening. The progression of lesions into the critical illness may manifest multiple diffusive consolidations in both lungs, appearing "white lungs" with a small amount of pleural effusion. Manifestations in the absorption stage include dissipation or decreased density of the previous lesions, or evolution into fiber or cord-like opacities. Therefore, DR is suitable for only primary hospitals without CT, or for severe or critically severe patients [34].

It has been reported that abnormal CT images may occur in $60 \%$ to $93 \%$ of the cases prior to a positive nucleic acid antibody test (or both occur simultaneously) [35]. The sensitivity of chest CT is greater than that of RT-PCR, which supports the use of chest CT to screen for COVD-19, especially when patients consistent with clinical and epidemiological profiles of COVD-19 but negative in RT-PCR tests are screened [36]. Therefore, chest CT scans with an appropriate scanning plan and parameters for COVID-19 patients will have a synergistic diagnostic effect in evaluating patients. The present consensus about COVID-19 among Chinese radiological experts is that high-resolution CT (HRCT) should be the main modality in screening and diagnosing this disease, with 16 layers or above, routine dosage, plain scans, a reconstruction layer thickness of not more than $3 \mathrm{~mm}$ and a layer thickness of $1 \mathrm{~mm}$ [37]. 


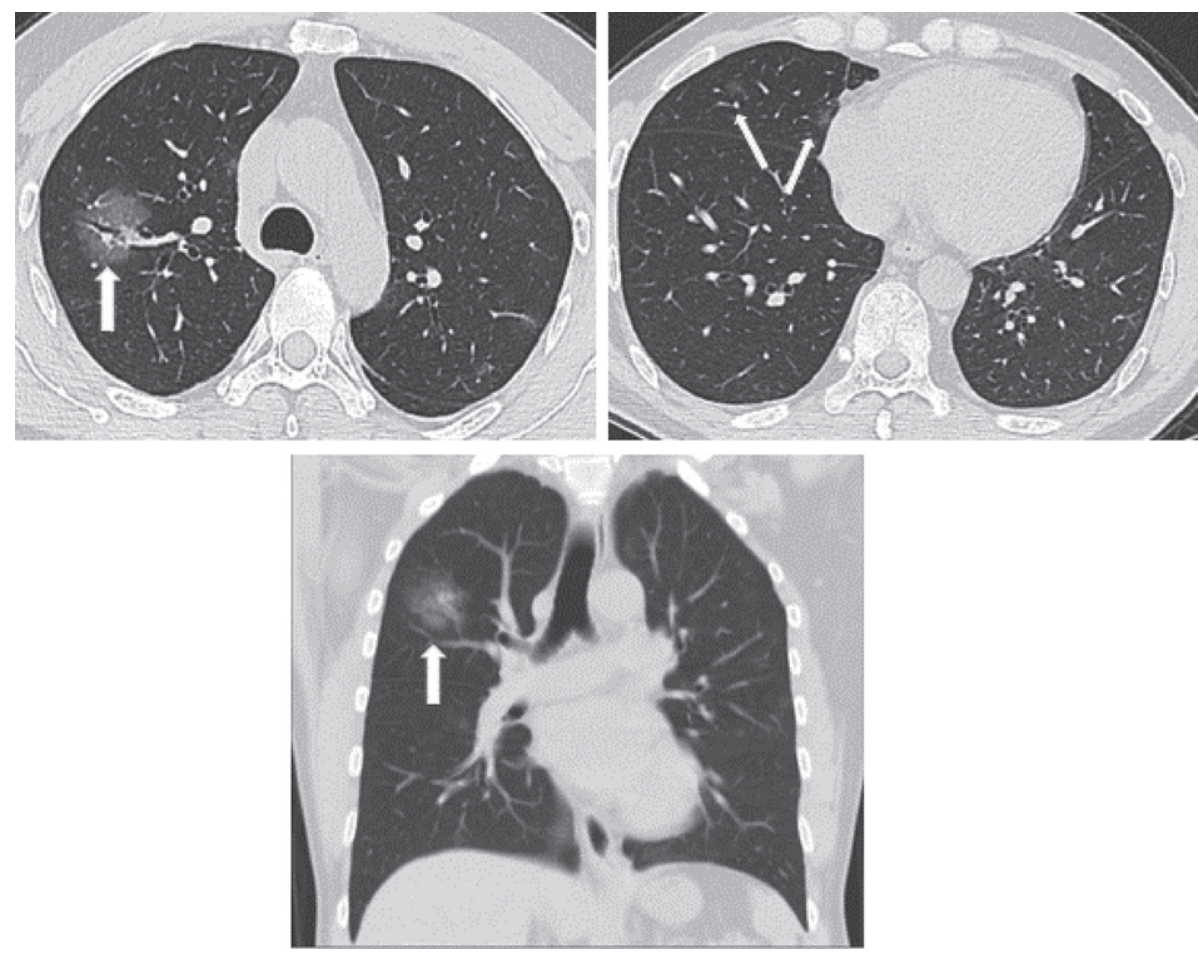

Fig. 1. Example images show a 52-year male patient who had onset of fever up to $39.1^{\circ}$ on January 26th, 2020, complicated with fatigue and dry cough with a positive nucleic acid test. He underwent the first CT scan on January 27th, 2020, day 2 after the onset, which showed in the upper right lung a patchy GGO containing thickened blood vessels and air bronchogram. Small patches of GGOs are scattered in the middle lobe of the right lung.

The CT manifestations of COVID-19 depend on a patient's age, immune status, disease stage at the time of scanning, underlying diseases, and drug intervention. Main radiological features include: (1) distribution: dominantly in peripheral pulmonary/subpleural bands, and distributed along bronchovascular bundles, (2) quantity: mostly three or more lesions, and rarely individual or pairs, (3) shape: patchy, bulky, or nodular, or in a shape of blocks, cellular texture, or reticular, (4) density: mostly uneven, manifesting GGOs, signs of paving stone, thickened interlobular septum with consolidation, and thickened bronchial wall, and (5) various complicated symptoms, but rare signs of pleural effusion or mediastinal lymphadenopathy.

According to guidelines issued by the Radiology Branch of the Chinese Medical Association (Imaging Diagnostic Guideline for Novel Coronavirus Pneumonia - Edition 2020), the Infectious Disease Group of the Chinese Society of Radiology (Adjuvant Imaging Diagnostic Guideline for Novel Coronavirus Pneumonia), the Project Group of Prevention and Control of Novel Coronavirus Pneumonia in Zhongnan Hospital of Wuhan University (Rapid Guideline for Diagnosis and Treatment of Novel Coronavirus Pneumonia (SARS-CoV-2)), as well as domestic and international literature, CT manifestations for COVID-19 can be classified into three stages. Specifically, based on the onset timepoint and the body's response to the virus, manifestations can be classified into the following three stages:

\subsection{Early stage}

This stage lasts 1-3 days after the onset of clinical manifestations, including fever, and dry cough. The pathological changes in this stage include dilation and congestion of alveolar septal capillaries, exudate in alveolar cavity, and interstitial edema in interlobular septum. CT scans manifest single 

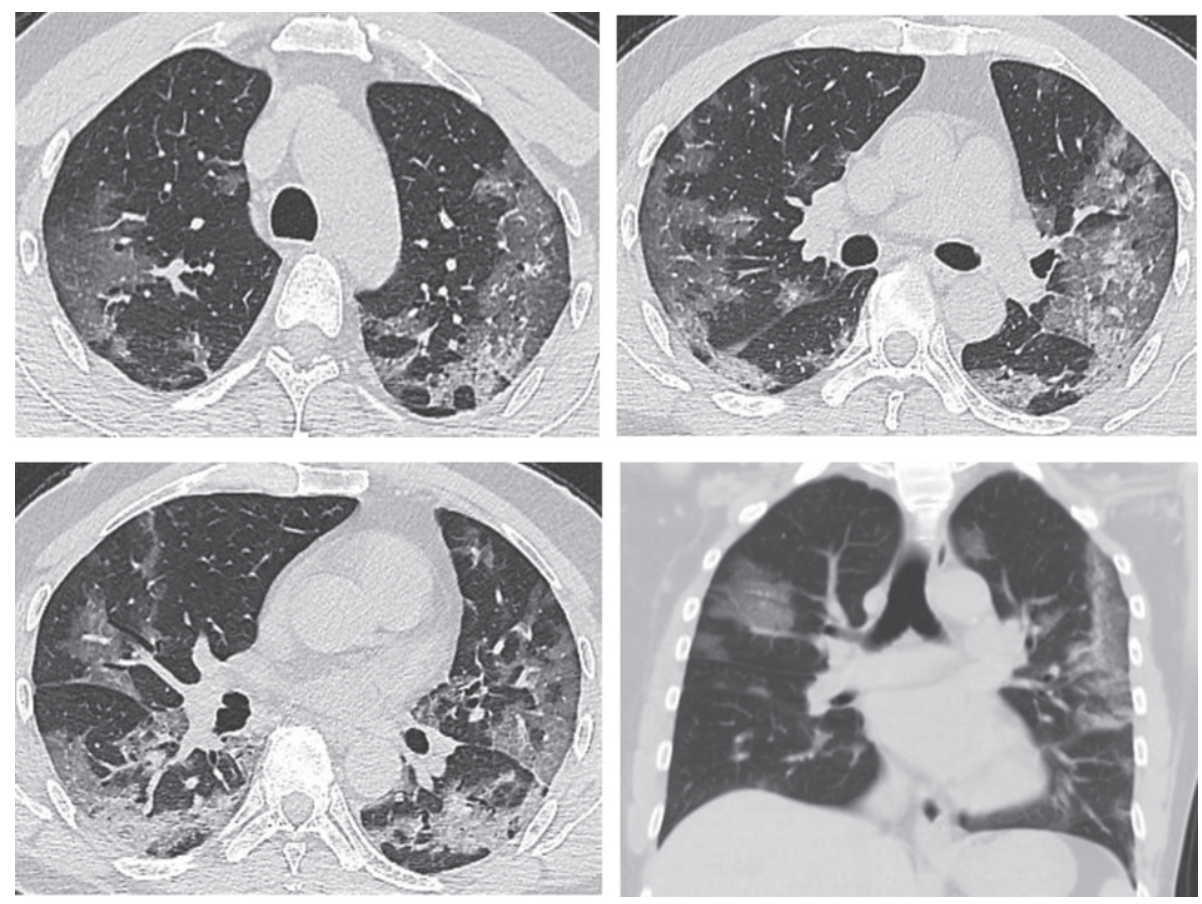

Fig. 2. Images of the same patient as shown by Fig. 1, which manifest an obviously increased number of lesions with progression in both lungs in CT scans on February 3rd, 2020, day 9 after the onset. Multiple large patchy GGOs appear in both lungs with consolidations containing air bronchogram inside and obvious thickening of interlobular septum.

or multiple scattered patchy or bulky GGOs, separated by lobular septum in honeycomb or reticular thickening [38-44] (Fig. 1).

Generally, lesions in the early stage do not involve an entire pulmonary section. A patient without any other pulmonary disease will not show lymphadenectasis in the mediastinum or hilum, pleural thickening, or pleural effusion. The pathological changes are considered to be correlated with pathological mechanisms including the vulnerability of terminal bronchioles and the pulmonary parenchyma around the respiratory bronchioles for viral pneumonia in the early stage, subsequent involvement of the whole pulmonary lobule, and diffusive lobular injuries.

\subsection{Progressive stage}

This stage refers to day 3-14 after the onset of clinical manifestations. The pathological feature during this stage is that the interstitial vascular dilation seems more obvious than before, and the exudate containing a large number of cells accumulates in the alveolus cavity, both of which will further aggravate the alveolus and interstitial edema. Cellulosic exudation enters every alveolus through intercellular connection to form a fusion state. CT scans show faded GGOs and consolidations appearing more distributed in comparison with the early stage and maintaining the dominance in peripheral pulmonary/subpleural distribution, with enlargement and fusion of some lesions invading subsequently multiple pulmonary lobes. The lesions are irregular, wedge-shaped or fan-shaped, with unclear borders, scattered in multiple foci or even diffuse, showing bilateral asymmetry. Opacities of soft tissues are observed, including broncho-vascular bundle thickening or subpleural multifocal pulmonary consolidation, showing rapid progression and changes, with great morphological variation within short-term reexamination. This can be complicated with tissue necrosis to form small cavities, and air bronchogram is commonly seen. It may also be accompanied by thickening of interlobular septum, manifesting 

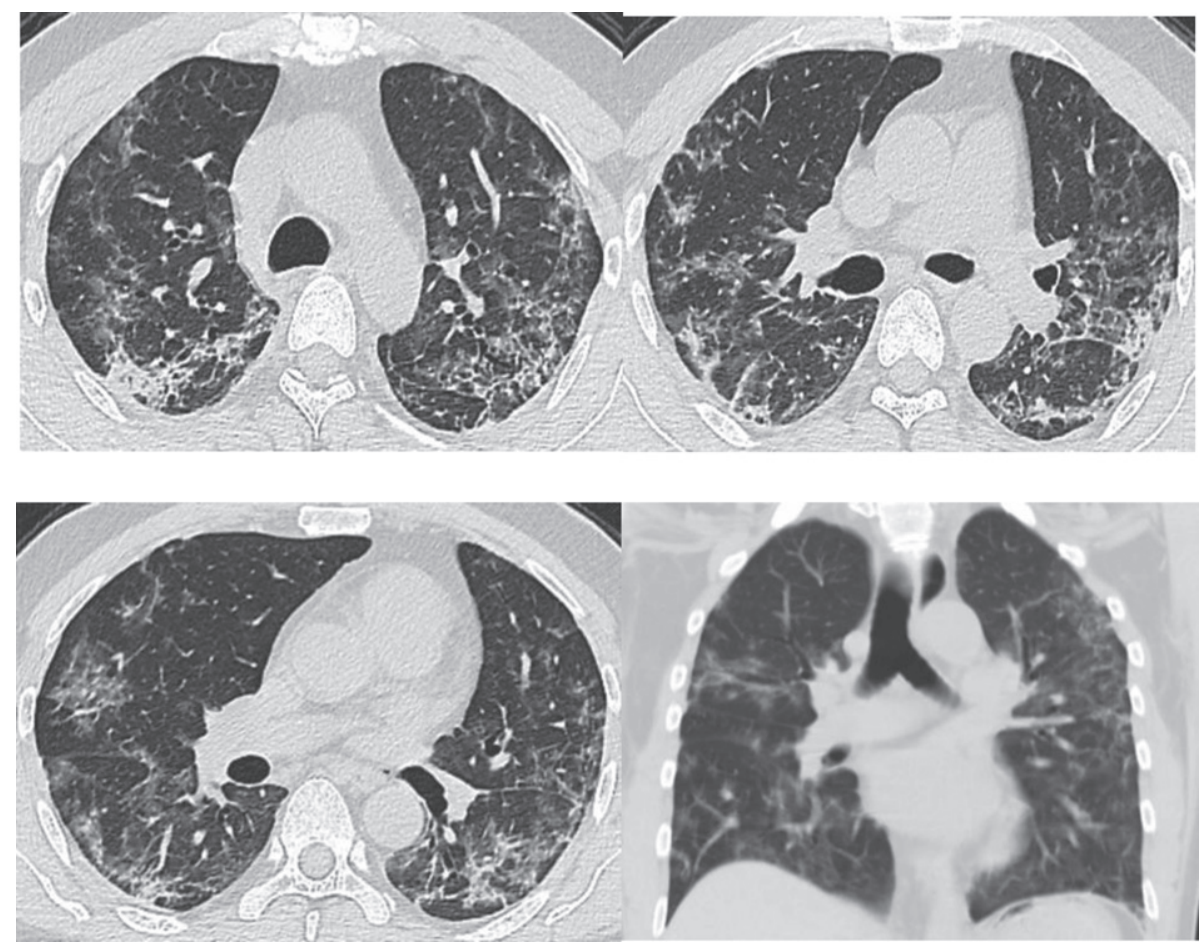

Fig. 3. Images of the same patient as shown by Figs. 1 and 2. CT scans on February 21st, 2020, day 24 after onset (during the absorption stage), show a gradual decrease and absorption of large patches of GGOs in both lungs, which are scattered with cord-like, reticulate and patchy opacities, with obvious thickening of interlobular septum.

"paving stone" sign, or complicated with fibrotic focus. Some cases manifest GGOs, usually without pleural effusion. Very few cases are complicated with lymphadenectasis in mediastinum and hilum. The disease in the progression stage often evolves abruptly and needs vigorous treatment. One should be alert of the occurrence of acute respiratory distress syndrome (ARDS) (Fig. 2).

If the patient does not receive effective treatment in time, pulmonary consolidation may occur on basis of the existing GGOs, in addition to the enlargement of GGOs and increase of their number. The main pathological feature in this stage is the cellulosic exudation in alveolar cavities and the gradual disappearance of capillary hyperemia of alveolar walls. Diffusive lesions in both lungs are observed in CT scans, and a few patients manifest "white lungs" $[45,46]$. The lesions may increase by $50 \%$ in their sizes within 48 hours, dominated by consolidations and complicated with GGO, air bronchogram, and multiple cord-like opacities, which is called clinically severe pneumonia and may develop clinically into the critically severe type if the disease keeps progressing.

\subsection{Absorption stage}

This stage refers to the 2-3 weeks' time period after the onset of clinical symptoms. The area of lesions shrinks after effective treatment. CT scans manifest patchy consolidations or cord-like opacities. The exudates undergo absorption or organization by the body over time with decreased density, and the pulmonary consolidations dissipate gradually. Along with the gradual absorption of the lesions, reticulate interlobular septal thickening is observable, with thickened and twisted bronchial walls in the image of cord-like changes, and a few scattered patchy high-density opacities being visible [38-46] (Fig. 3). 

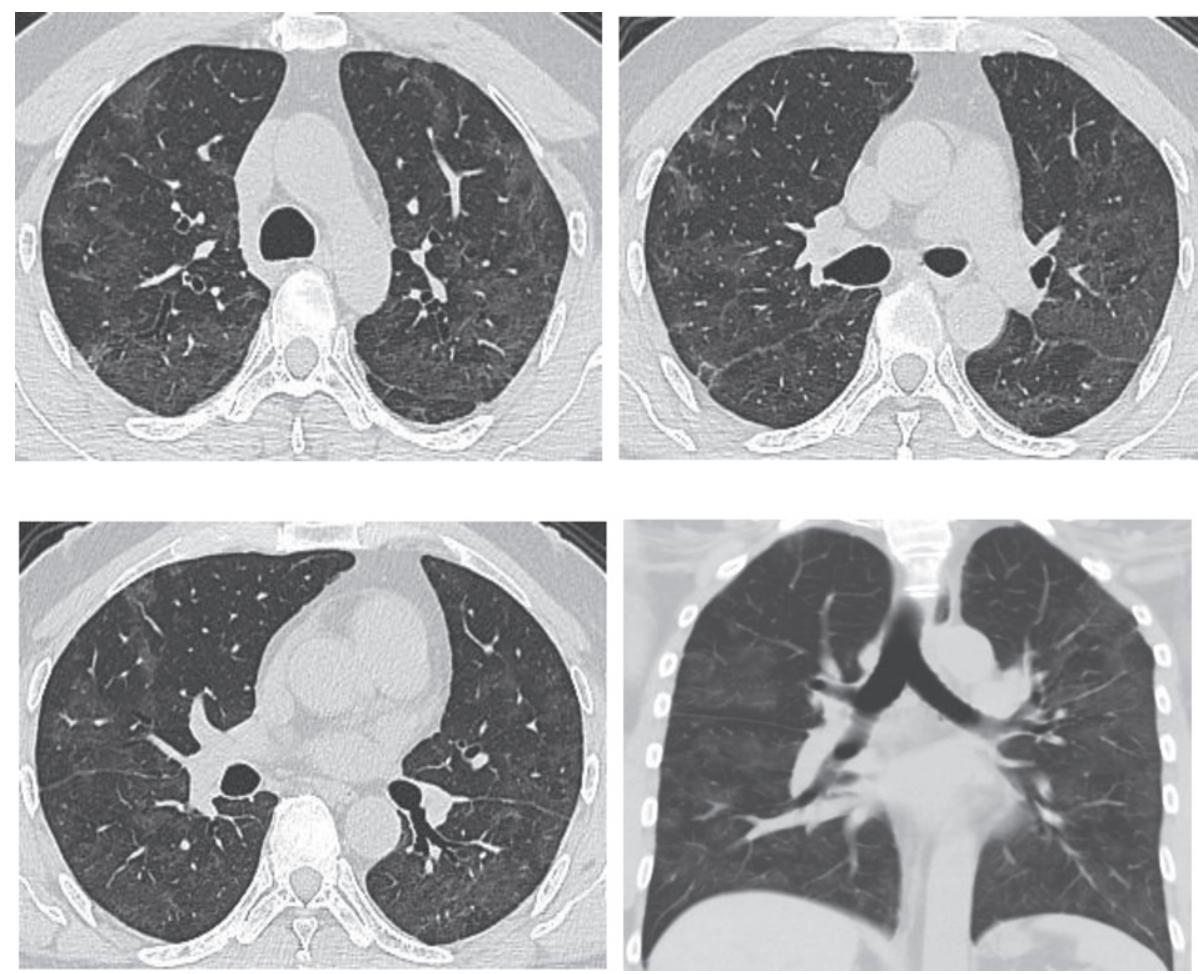

Fig. 4. Chest CT scans on March 20th, 2020, for the same patient shown by Figs. 1-3, after treatment, showing obvious absorption of lesions (decreased lesions with partial fibrosis). After admission, the patient received antiviral and atomizing therapies, but symptoms aggravated with repeated fever, cough, expectoration, and gasp. A written notice of danger was given on February 2nd 2020, and the patient was given oxygen inhalation, continuous ECG monitoring, finger pulse oxygen monitoring, anti-infection treatment, anti-virus therapy, atomization of recombinant human interferon $\alpha 2 b$ and intravenous injection of human immunoglobulin to improve immunity, as well as symptomatic treatments to reduce inflammation. The symptoms improved after treatment. Chest CT scans on March 20th showed the obvious absorption of the lesions. Re-test of nucleic acid of novel coronavirus after an interval of 24 hours was negative. The patient was discharged in agreement after consulting with the Municipal Novel Coronavirus Pneumonia Expert Group of the 4th People's Hospital of Nanning City.

The patient of Fig. 3 had a follow-up chest CT examination four weeks later. The images showed that the patient's symptoms significantly improved after treatment with the obvious absorption of lesions (Fig. 4).

The characteristic feature of COVID-19 is GGO, which is a radiological concept manifesting a minor increase of density and a cloudy opacity, but with observable textures of blood vessels and bronchi inside [47]. GGO is a non-specific term and highly suggests that the disease of lung tissue is active or reversible. Lesions of GGOs in lungs of patients with COVID-19 decrease gradually over time, while fibrous cord-like opacities increase gradually, which becomes the most common radiological manifestation. Research has shown that the most obvious changes of intrapulmonary lesions are observable during day 6-9 after admission in $75.0 \%$ of the patients, and relatively obvious absorption can be seen during day 10-14 of the hospital stay in $76.9 \%$ of the patients [48]. In addition, some research work implies that the dandelion fruit sign is the characteristic change in COVID-19 patients [49]. The longer the duration of a SARS-CoV-2 infection, the more changes can be seen in CT images [50]. The radiological changes in late sequela remain unknown [51]. 

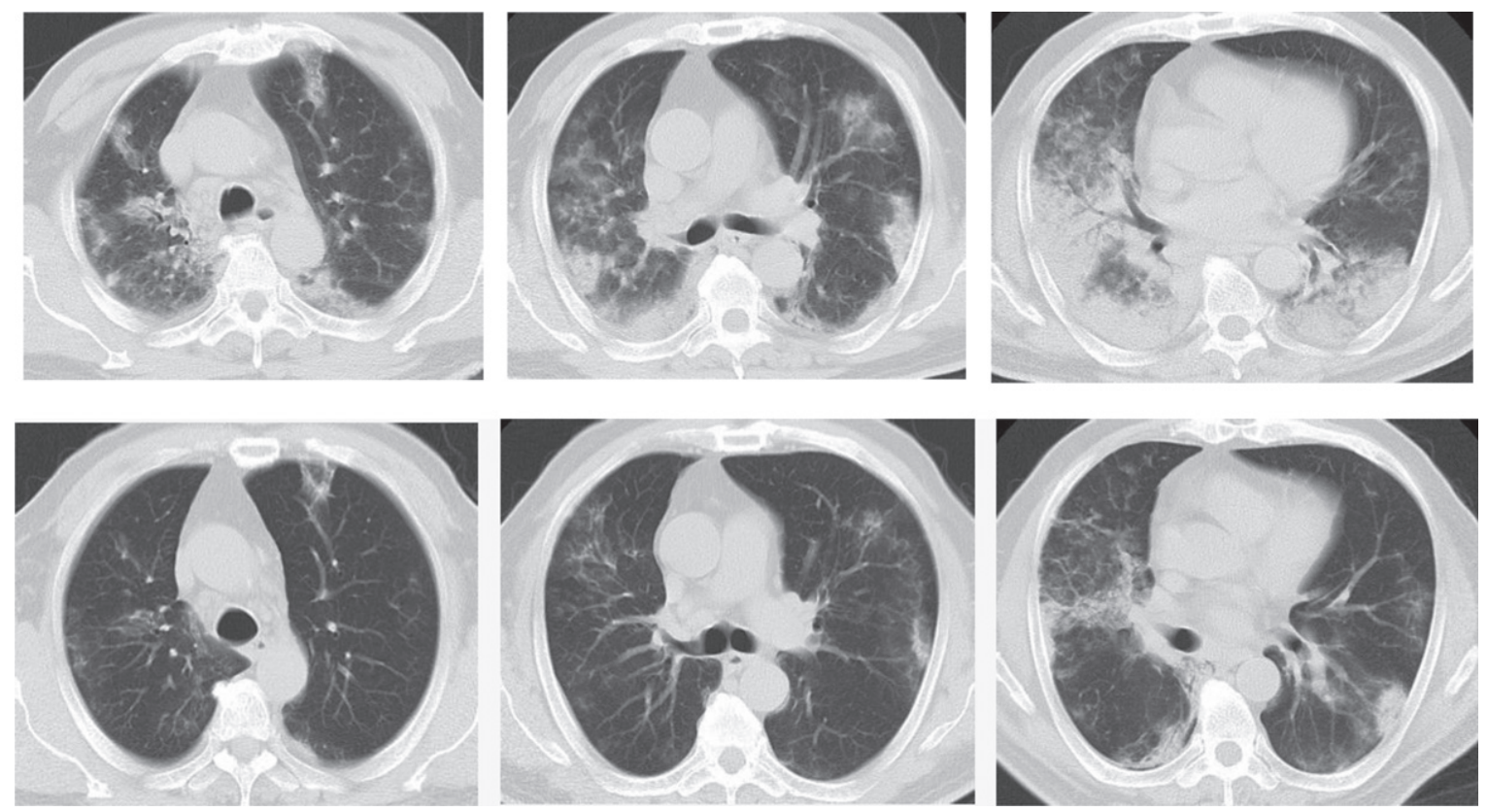

Fig. 5. Example CT images of a 63-year-old male patient diagnosed with H7N9 avian pneumonia. Top row shows chest CT scans taken on January 17th, 2014, day 11 after the onset, in the progression phase. CT scans show patches of ground glass opacities in the upper right lung and the upper left lung, dominantly distributed in the subpleural zone. Consolidations can be seen in the lower lobes of both lungs, with air bronchogram inside dominantly at the lower right lung. Bottom row shows chest CT taken on January 24th, 2014, day 18 after the onset during the absorption period. CT shows the obvious absorption of ground glass opacities and consolidations in both lungs, which have been dominantly interstitial changes and small patches of consolidations.

\section{Differential diagnosis}

\subsection{Differentiation between COVID-19 pneumonia and other viral pneumonias}

Other viral pneumonia manifest generally diffuse large patches of GGOs in both lungs with thickening of interlobular septum, which makes it hard to differentiate them from COVID-19 pneumonia in images and clinically. Definite epidemic history provides suggestive efficacy for differentiation for all kinds of viral pneumonias, especially COVID-19, while confirmed diagnosis should be based on etiological detection.

\subsubsection{Differentiation with pneumonia induced by SARS and MERS}

Pathogens for SARS, MERS, and COVID-19 all belong to the coronavirus family, with similar pathogenic mechanisms among them all. Given the diversity in imaging, without obvious specificity, it is hard to differentiate merely based on imaging. The incorporation of epidemic features and pathogenic tests may facilitate the discrimination.

\subsubsection{Differentiation with pneumonia by avian influenza virus}

Avian influenza virus pneumonia in human is an infectious disease of the acute respiratory tract induced by several subtypes of the avian influenza virus such as H7N9 and H5N1. It spreads mainly through contact with poultry, in contrast to the contamination through wild animals for the novel coronavirus pneumonia showing an obvious human-human transmission. As for imaging, lesions seem wider in lungs for avian influenza pneumonia in human with severe pulmonary consolidation, although 
both pneumonias manifest patches or large sheets of ground glass opacities and consolidations in the lung without major difference (Fig. 5). Therefore, some difficulties exist in differentiating them.

\subsection{Differentiation with mycoplasma pneumonia}

Mycoplasma pneumonia, observed commonly in children and young people, is characterized clinically by paroxysmal and irritant dry cough and by the chest X-ray showing either interstitial infiltration (e.g., increased, thickened or gridded lung markings) or consolidations of segments or lobes manifesting patchy or fan-shaped infiltration. Imaging of CT scans is characterized by thickening of the bronchial wall, centrilobular nodules, consolidations distributed along lobes, segments or subsegments of the lung, and enlargement of mediastinal lymph nodes. In comparison, enlarged mediastinal lymph nodes are rarely seen in novel coronavirus pneumonia. Positiveness in mycoplasma antibody can be observed in laboratory tests, as a response to Macrolides antibiotics. A differentiation can be made, therefore, based on clinical features and serological examination.

\subsection{Differentiation between COVID-19 pneumonia and bacterial pneumonia}

Bacterial pneumonia often manifests shivering, high fever, and bloody or rusty expectoration, with laboratory tests showing an increase of leukocytes and neutrophils. Imaging usually shows small patches of consolidations distributed along the bronchi and even fused into the distribution along subsegments, segments, or lobes, which show good response to antibiotics and are easily differentiated with COVID-19 pneumonia.

\section{Summary}

Currently, detection of viral nucleic acid is still the gold standard for diagnosing COVID-19 [52], although it has high specificity but low sensitivity. Temporal difference exists in clinical manifestations, between nucleic acid detection and CT imaging during the early stage of COVID-19, which might be the critical factor for misdiagnosing the disease, and a factor for transmission of the disease [53]. A cross validation should be performed by clinicians, based on clinical manifestations and CT imaging, to make the clinical diagnosis of COVID.

It is also very likely that outbreaks of new diseases caused by coronavirus might occur in the future, given our limited understanding of SARS-CoV-2, climate and ecological changes possibly affecting the course of outbreaks, and continuing interactions between humans and animals. Therefore, future research should focus on developing effective drugs and vaccines through strengthened international cooperation to investigate and contain infectious diseases caused by coronavirus as a vital public health issue [54].

\section{Funding Acknowledgments}

This study was partially funded by Shenzhen Science and Technology Program (Grant No. KQTD2017033110081833), as well as partially supported by the Intramural Research Program of the Lister Hill National Center for Biomedical Communications (LHNCBC), the U.S. National Library of Medicine (NLM), and the National Institutes of Health (NIH). 


\section{References}

[1] C. Wang, et al., A novel coronavirus outbreak of global health concern, Lancet 395(10223) (2020), 470-473.

[2] National Health Commission. An Announcement by National Health Commission of People's Republic of China (No. 1 in 2020) [EB/OL]. [2020-01-21] [2020-04-06]. http://www.gov.cn/xinwen/2020-01/21/content_5471158.htm.

[3] World Health Organization (WHO). Summary table of SARS cases by country, 1 November 2002 - 7 August 2003[EB/OL]. [2003-08-15] [2020-04-06]. https://www.who.int/csr/sars/country/2003_08_15/en/.

[4] N.S. Chen, et al., Epidemiological and clinical characteristics of 99 cases of 2019 novel coronavirus pneumonia in Wuhan, China: A descriptive study, Lancet 395(10223) (2020), 507-513.

[5] Y. Chen, et al., Emerging Coronaviruses: genome structure, replication, and pathogenesis, J Med Virol 92(4) (2020), $418-423$.

[6] X. Xu, et al., Evolution of the novel coronavirus from the ongoing Wuhan outbreak and modeling of its spike protein for risk of human transmission, Sci China Life Sci 63(3) (2020), 457-460.

[7] Y.Z. Zhou, et al., Analysis of the variation and evolution of coronavirus SARS CoV-2, Journal of Southern Medical University 40(02) (2020), 152-158.

[8] S. Perlman, Another decade, another coronavirus, N Engl J Med 382(8) (2020), 760-762.

[9] South China Agricultural University. Press conference of research on novel coronavirus pneumonia epidemic in Guangzhou, February 7th, 2020.

[10] Expert Team of Control of Novel Coronavirus Pneumonia under Chinese Society of Preventive Medicine, The latest knowledge of epidemiology of novel coronavirus pneumonia, Chinese Journal of Viral Diseases (2020), 1-7. https://doi.org/10.16505/j.2095-0136.2020.0015.

[11] X.L. Tang, et al., On the origin and continuing evolution of SARS-CoV-2, National Science Review, nwaa036, https://doi.org/10.1093/nsr/nwaa036.

[12] J. G. de Jesus, et al, First cases of coronavirus disease (COVID-19) in Brazil, South America Virological, 2020. http://virological.org/t/first-cases-of-coronavirus-disease-covid-19-in-brazil-south-america-2-genomes3rd-march-2020/409

[13] Z. Ma, G.J. Cao, M. Guan, Status and progress in researches on human coronavirus, International Journal of Laboratory Medicine 41(05) (2020), 518-522.

[14] Notification of printing and distributing therapeutic regimens for novel coronavirus pneumonia (Trial Version VII), General Office of National Health Commission, and Office of State Administration of Traditional Chinese Medicine. [2020-03-03] [2020-04-06]. http://www.gov.cn/zhengce/zhengceku/2020-03/04/content_5486705.htm.

[15] Y. Gong, et al., Present status in researches on coronavirus, Chinese Journal of Bioengineering 40(Z1) (2020), 1-20.

[16] B.L. Xu, et al., Progress in researches on novel coronavirus COVID-19, Chinese Journal of Nosocomial Infection 30(6) (2020), 806-811.

[17] T.Y. Qiu, et al., Identification of potential cross-protective epitope between 2019-nCoV and SARS virus, J Genet Genomics 47(2) (2020), 115-117.

[18] F. Li, Structure, function, and evolution of coronavirus spike proteins, Annu Rev Virol 3(1) (2016), $237-261$.

[19] Epidemiology Team in Emergency Response Mechanism for Novel Corona virus Pneumonia in Chinese Center for Disease Control and Prevention, Analysis of epidemiological features of novel coronavirus pneumonia, Chinese Journal of Epidemiology 41(2) (2020), 145-151.

[20] W.J. Guan, et al., Clinical characteristics of 2019 novel coronavirus infection in China. NEJM. Published online February 28, 2020. DOI: 10.1056/NEJMoa2002032.

[21] Y.J. Zhuang, et al., Clinical and epidemiological characteristics of 26 confirmed cases of novel coronavirus pneumonia, Chinese Journal of Nosocomial Infection 30(6) (2020), 817-820.

[22] Y.H. Jin, et al., A rapid advice guideline for the diagnosis and treatment of 2019 novel coronavirus (2019-nCoV) infected pneumonia (standard version), Mil Med Res 7(1) (2020), 4.

[23] R.C. Wang, et al., Emergence of SARS-like Corona virus poses new challenge in China, Journal of Infection 80(3) (2020), 350-371.

[24] V.J. Munster, et al., A Novel Corona virus Emerging in China - Key Questions for Impact Assessment, $N$ Engl J Med 382(8) (2020), 692-694.

[25] H.S. Shi, et al., Clinical features and imaging findings of novel coronavirus (2019-nCoV) pneumonia, Clinical Radiology 39(01) (2020), 8-11.

[26] V.M. Corman, et al., Detection of 2019 novel coronavirus (2019-nCoV) by real-time RT-PCR. Euro Surveill 25(3) (2020), 2000045.

[27] F. Wu, et al., A new coronavirus associated with human respiratory disease in China, Nature 579(7798) (2020), $265-269$. 
[28] Notice of Prevention and Control Plan for Novel Corona virus Pneumonia issued by the General Office of National Health Committee of People's Republic of China (Fifth Edition) [EB/OL]. [2020-02-21] [2020-04-06]. http://www.nhc.gov.cn/jkj/s3577/202002/a5d6f7b8c48c451c87dba14889b30147.shtml.

[29] W. Da, et al., Misunderstanding of nucleic acid testing for 2019-nCoV, Chinese Journal of Nosocomiology 30(8) (2020), 1153-1156.

[30] Z. Xu, et al., Pathological findings of COVID-19 associated with acute respiratory distress syndrome, Lancet Respir Med 8(4) (2020), 420-422.

[31] D. Wang, et al., Clinical Characteristics of 138 Hospitalized Patients with 2019 Novel Corona virus-Infected Pneumonia in Wuhan, China, JAMA 323(11) (2020), 1061-1069.

[32] J.W. Tang, et al., Emergence of a novel coronavirus causing respiratory illness from Wuhan, China, J Infect 80(3) (2020), 350-371.

[33] Q. Liu, et al., Report of gross findings of systemic anatomy in autopsy of a death due to novel coronavirus pneumonia, Journal of Forensic Medicine 36(1) (2020), 19-21.

[34] Infectious Diseases Group of Radiology Branch of Chinese Medical Association, A radiological diagnostic guideline for novel coronavirus pneumonia Version I, 2020, 2020-1-24.

[35] T. Ai, et al., Correlation of Chest CT and RT-PCR Testing in Corona Virus Disease 2019 (COVID-19) in China: A Report of 1014 Cases, Radiology, 2020, 200642.

[36] Y.C. Fang, et al., Sensitivity of Chest CT for COVID-19: Comparison to RT-PCR, Radiology, $2020,200432$.

[37] H.X. Guan, et al., Preliminary study of clinical imaging features of 2019 novel coronavirus (2019-nCoV) pneumonia, Radiological Practice (2020), 1-6. https://doi.org/10.13609/j.cnki.1000-0313.2020.02.001.

[38] Professional Committee of Infection and Inflammation Radiology of Chinese Research Hospital Association, Guideline for adjuvant imaging diagnosis for novel coronavirus pneumonia, Chinese Medical Imaging Technology 36(3) (2020), $1-11$.

[39] Radiology Branch of Chinese Medical Association, Radiological diagnosis of novel coronal pneumonia: Expert Recommendation by Radiology Branch of Chinese Medical Association (Version I), Chinese Journal of Radiology (2020), 54. DOI: 10.3760/cma.j.issn.1005-1201.2020.0001.

[40] Prevention and Control Group of Novel Corona virus Pneumonia of Zhongnan Hospital of Wuhan University) EvidenceBased Medicine Branch of China Healthcare Association for International Exchange and Promotion, Rapid guideline for diagnosis and treatment of novel coronavirus pneumonia (2019-nCoV) 2020-02-02.

[41] Q. Liang, Imaging examination, diagnosis and prevention and control of nosocomial infection in patients with novel coronavirus pneumonia: Consensus of radiologists in Hunan Province, Journal of Central South University (Medical Version) 45(03) (2020), 221-228.

[42] C.Y. Liu, et al., A comparative study on CT findings between novel coronavirus pneumonia manifesting groundglass opacities and early-stage lung tumor, Chinese Journal of Thoracic and Cardiovascular Surgery 27(04) (2020), 376-380.

[43] X.M. Gong, et al., Preliminary discussion of CT findings of novel coronavirus pneumonia (COVID-19), Radiological Practice 35(03) (2020), 261-265.

[44] F.M. Liu, et al., Chest CT findings and clinical features of novel coronavirus pneumonia (COVID-19), Radiological Practice 35(03) (2020), 266-268.

[45] K. Wang, et al., An analysis on features of chest CT findings of novel coronavirus pneumonia, Chinese Clinical Medicine 27(01) (2020), 27-31.

[46] G.Z. Fu, et al., The application of chest CT scans to screening of novel coronavirus pneumonia, Journal of Wenzhou Medical University (2020), 1-9. http://kns.cnki.net/kcms/detail/33.1386.r.20200219.1451.002.html.

[47] K. Ikeda, et al., Differential diagnosis of ground-glass opacity nodules: CT number analysis by three dimensional computerized quantification, Chest 132(3) (2007), 984-990.

[48] J.C. Wang, et al., Dynamic changes of chest CT findings in patients with 2019 coronavirus disease (COVID19), Journal of Zhejiang University (Medical Version) (2020), 1-13. http://kns.cnki.net/kcms/detail/33.1248. R.20200225.1528.004.html.

[49] X.B. Fu, et al., Dandelion fruit sign: a CT sign for diagnosing novel coronavirus pneumonia, Journal of Southern Medical University (2020), 1-5. http://kns.cnki.net/kcms/detail/44.1627.R.20200228.1823.004.html.

[50] A. Bernheim, et al., Chest CT Findings in Corona virus Disease-19 (COVID-19): Relationship to Duration of Infection, Radiology 2020, 200463. doi:10.1148/radiol.2020200463.

[51] J. Chen, et al., An analysis on clinical features of 29 cases of 2019 novel coronavirus pneumonia, Chinese Journal of Tuberculosis and Respiration 43 (2020), E005.

[52] Disease Prevention and Control Bureau of the National Health Commission. Prevention and control plan for novel coronavirus pneumonia (Version II) (2020), 1-22. 
[53] Y.H. Du, et al., Preliminary discussion on clinical features and CT findings in early stage of family cluster of novel coronavirus pneumonia, Journal of Xi'an Jiaotong University (Medical Version) 41(02) (2020), 215.

[54] R. Min, et al., Progress in researches on clinical features and pathogenesis of novel coronavirus pneumonia, Chinese Journal of Nosocomial Infection 30(8) (2020), 1136-1141. 